\title{
Thrombophlébite cérébrale en post-partum retardé
}

\section{Cerebral Venous Thrombosis in Late Postpartum}

\section{P. Le Borgne • M. Mihalcea-Danciu • C. Kam}

Reçu le 5 janvier 2015; accepté le 13 mars 2015

(C) SFMU et Lavoisier SAS 2015

Une femme de 34 ans se présente aux urgences pour céphalée et trouble du comportement évoluant depuis une semaine. La patiente ne présente aucun antécédent notable, elle vient néanmoins d'accoucher sans complication de son premier enfant dix semaines auparavant. Le post-partum immédiat a été compliqué d'un baby-blues régressif. Son mari qui l'accompagne évoque des hallucinations visuelles la veille, le médecin traitant adresse la patiente pour probable psychose puerpérale confuso-délirante. L'examen clinique initial retrouve une patiente asthénique, peu réactive, quasi mutique. Elle décrit des céphalées occipitales intermittentes mais aucun signe de localisation neurologique n'est objectivé. La patiente bénéficie d'un angioscanner veineux retrouvant une thrombophlébite cérébrale du sinus longitudinale supérieure avec signe du delta (Fig. 1). Le signe du delta (empty-delta sign) apparaît comme une aire hypodense entourée d'une prise contraste (correspondant au thrombus et aux veines collatérales dilatées). La grossesse et le postpartum augmente le risque d'événement thrombotique (hypercoagulabilité). L'incidence moyenne des thrombophlébites cérébrales en post-partum est de 12 cas pour 100000 accouchements [1]. Elles surviennent habituellement précocement après l'accouchement entre le $4^{\mathrm{e}}$ et le $21^{\mathrm{e}}$ jours. Mais une étude récente semble démontrer le risque accru d'événement thromboembolique veineux, également entre 6 et 12 semaines de post-partum [2]. Les signes cliniques aspécifiques rendent le diagnostic difficile, d'autant qu'il n'existe aucune corrélation clinicotopographique. Il est

P. Le Borgne $(\bowtie) \cdot$ M. Mihalcea-Danciu $\cdot$ C. Kam Service d'accueil des urgences, CHU de Strasbourg, hôpital de Hautepierre, 1 avenue Molière,

F-67098 Strasbourg, France

e-mail : pierrick_med@yahoo.fr

P. Le Borgne

Faculté de médecine, Université de Strasbourg,

4 Rue Kirschleger, F-67000 Strasbourg

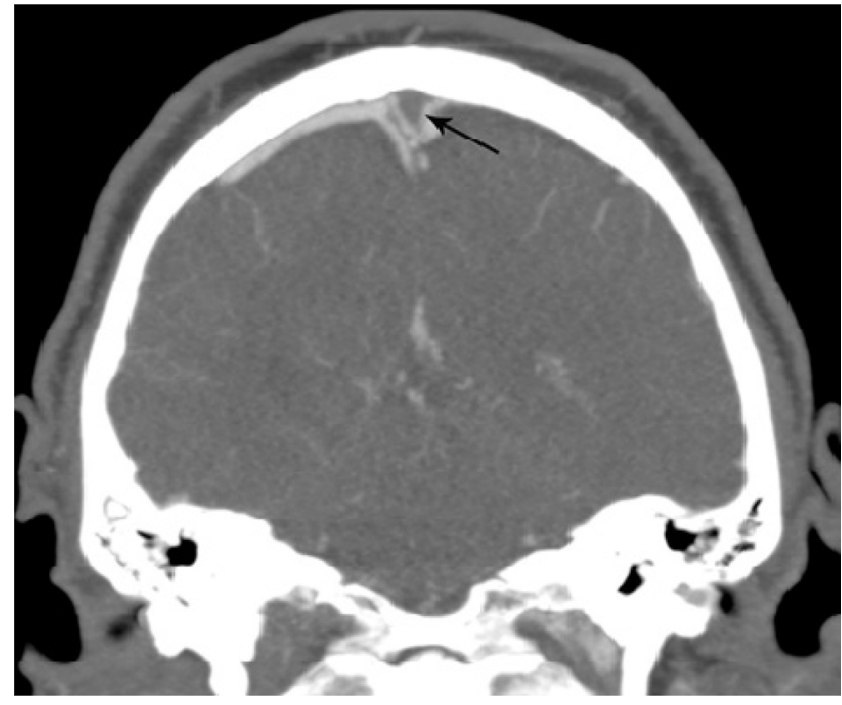

Fig. 1 Angioscanner cérébral (temps veineux), coupe frontale. Flèche : signe du delta, thrombophlébite cérébrale du sinus longitudinale supérieure

important d'évoquer systématiquement ce diagnostic en post-partum puisqu'il s'agit de l'affection cérébrovasculaire la plus fréquente de cette période à risque thrombotique. $\mathrm{La}$ patiente a bénéficié d'une héparinothérapie initiale avec relais précoce par antivitamine $\mathrm{K}$ et est sortie sans séquelle. Le bilan de thrombophilie a par ailleurs retrouvé une mutation $\mathrm{du}$ facteur $\mathrm{V}$ Leyden (autre facteur de risque de thrombose).

\section{Références}

1. Saposnik G, Barinagarrementeria F, Brown RD, et al (2011) Diagnosis and management of cerebral venous thrombosis: a statement for healthcare professionals from the American Heart Association/ American Stroke Association. Stroke 42:1158-92

2. Kamel H, Navi BB, Sriram N, et al (2014) Risk of thrombotic event after the 6-week postpartum period. $\mathrm{N}$ Engl J Med 370:1307-15 